\title{
De novo transcriptomic analysis of cowpea (Vigna unguiculata L. Walp.) for genic SSR marker development
}

\author{
Honglin Chen, Lixia Wang, Xiaoyan Liu, Liangliang Hu, Suhua Wang and Xuzhen Cheng ${ }^{*}$ (D)
}

\begin{abstract}
Background: Cowpea [Vigna unguiculata (L.) Walp.] is one of the most important legumes in tropical and semi-arid regions. However, there is relatively little genomic information available for genetic research on and breeding of cowpea. The objectives of this study were to analyse the cowpea transcriptome and develop genic molecular markers for future genetic studies of this genus.

Results: Approximately 54 million high-quality cDNA sequence reads were obtained from cowpea based on Illumina paired-end sequencing technology and were de novo assembled to generate 47,899 unigenes with an N50 length of $1534 \mathrm{bp}$. Sequence similarity analysis revealed 36,289 unigenes (75.8\%) with significant similarity to known proteins in the non-redundant (Nr) protein database, 23,471 unigenes (49.0\%) with BLAST hits in the Swiss-Prot database, and 20,654 unigenes (43.1\%) with high similarity in the Kyoto Encyclopedia of Genes and Genomes (KEGG) database. Further analysis identified 5560 simple sequence repeats (SSRs) as potential genic molecular markers. Validating a random set of 500 SSR markers yielded 54 polymorphic markers among 32 cowpea accessions.

Conclusions: This transcriptomic analysis of cowpea provided a valuable set of genomic data for characterizing genes with important agronomic traits in Vigna unguiculata and a new set of genic SSR markers for further genetic studies and breeding in cowpea and related Vigna species.
\end{abstract}

Keywords: Vigna unguiculata (L.) Walp, Transcriptome, De novo assembly, Functional annotation, Genic SSR

\section{Background}

Cowpea [Vigna unguiculata (L.) Walp.] is a diploid Vigna crop $(2 \mathrm{n}=2 \mathrm{x}=22)$, and its genome size is estimated to be $620 \mathrm{Mb}$ [1]. It is the most important legume and semi-arid crop in sub-Saharan Africa and other parts of the world [2,3] and is widely cultivated in Africa, Latin America, Southeast Asia, and southwestern regions of North America. Its global annual production is approximately 5.8 million tons, with a minimum of 11 million hectares planted [4]. Additionally, cowpea plays an important role in human nutrition and is a critical nutrition source for animals in the dry season in many countries. With its highly efficient nitrogen fixation during crop rotation with cereal crops,

\footnotetext{
* Correspondence: chengxuzhen@caas.cn

The National Key Facility for Crop Gene, Resources and Genetic

Improvement, Institute of Crop Science, Chinese Academy of Agricultural Sciences, Beijing 100081, China
}

this plant can be used to boost soil fertility. Moreover, cowpea is an important income source for farmers and grain traders [5].

Despite its importance, cowpea has received little attention from the research perspective and is less studied than other legumes, such as common bean, chickpea, pea, and soybean. Only a relatively small number of expressed sequence tags (ESTs) and genome sequences of cowpea are available in public databases [1], even though its genome size is relatively small among legume species. The number of microsatellite markers in cowpea is still far fewer than those reported for other legume species, and the genetic base of cowpea is narrow. Informative molecular markers are largely lacking in cowpea. Research on its genome, genetic mapping and molecular breeding has lagged behind those of other pulse crop species, including common bean (Phaseolus vulgaris) and soybean (Glycine. max). However, significant progress has been made in genomic 
research on Medicago truncatula [6], Lotus japonicas [7], soybean (Glycine max) [8], pigeonpea (Cajanus cajan) [9], chickpea (Cicer arietinum) [10], common bean (Phaseolus vulgaris) [11], mung bean (Vigna radiate) [12], and adzuki bean (Vigna unguiculata) [13]. Many ESTs and complete genome sequences of these other legume species are available.

Various molecular markers have been developed in cowpea, such as RFLP (restriction fragment length polymorphism) [14], RAPD (random-amplified polymorphic DNA) [15], and AFLP (amplified fragment length polymorphism) [16]. However, the developed markers are insufficient for an informative genetic study as they are neither highly polymorphic nor easy to analyse. Microsatellite (SSR) markers are usually more informative and have high polymorphism, and they are highly abundant and well distributed throughout the genome in various plants. These SSR markers are a powerful tool for genetic diversity analysis, map construction, quantitative trait locus (QTL) identification, and marker-assisted breeding, and they enhance genetic research in cowpea regarding the genetic improvement of important traits [17]. However, due to a single domestication event [18] and its inherent self-pollination systems [4], cowpea has narrow genetic variability (approximately 16\%). The available molecular markers for cowpea are limited, and polymorphic SSR markers in cowpea may be difficult to identify [18]. These issues have been a constraint on cowpea genotyping and other genetic studies. A total of 15 polymorphic SSR markers were obtained from 30 cowpea microsatellite primer pairs [19]. A total of 1071 SSRs were identified in 15,740 cowpea unigene sequences downloaded from the National Center for Biotechnology Information (NCBI), and 102 SSR markers were finally characterized and validated [20]. However, the reported SSR markers in cowpea are not sufficient for an informative genetic study, and more efficient RNA-Seq designs and marker discovery techniques are needed to enhance marker development in the future. Many SSR markers have been developed in common bean [21], chickpea [22], pigeonpea [23], soybean [24], rice bean [25], adzuki bean [26], and mung bean [27]. Thus, there is an urgent need to develop numerous SSR markers that can be used by breeders in various molecular breeding strategies to increase breeding efficiency [28].

Transcriptomic data from RNA-Seq allows the efficient identification of large numbers of genic molecular markers compared to the traditional approaches of microsatellite development. Transcriptomic data can also increase the possibility of developing microsatellites associated with functional genes. Previous studies have shown that EST sequences generated using transcriptome techniques are an effective source of SSR markers for legume crops $[21,25-27,29]$ and other crops [30, 31]. De novo assembly of transcriptome data generated by RNA-Seq will provide valuable genetic resources for further genomic studies and for developing genic SSRs for a crop with a low number of markers available for the species. This study aims to develop more genic SSR markers for cowpea through RNA-Seq technology, characterize the distribution of SSR motifs in the obtained sequences, and validate a set of genic SSR markers.

\section{Results}

Sequencing and de novo assembly

A total of 57,214,890 paired-end raw reads were generated by Illumina next-generation sequencing for the cowpea cultivar Zhongjiang No. 1. After quality filtering of the reads, 54,417,074 clean reads were obtained with a GC content of $46.7 \%$, and the Q20 percentage was over $98 \%$. These clean reads were assembled into 68,135 contigs and 47,899 unigenes, and the average length of the assembled unigenes was $871 \mathrm{bp}(\mathrm{N} 50=1534 \mathrm{bp})$. The sequences of the unigenes are listed in Additional file 1. The 68,135 contigs were composed of 48,337 contigs (70.9\%) within the length range from 201 to $500 \mathrm{bp}, 8774$ contigs (12.9\%) within the length range from 501 to $1000 \mathrm{bp}, 4923$ contigs (7.2\%) within the length range from 1001 to $1500 \mathrm{bp}$, 2906 contigs $(4.3 \%)$ within the length range from 1501 to $2000 \mathrm{bp}, 1523$ contigs (2.2\%) within the length range from 2001 to $2500 \mathrm{bp}$, and 760 contigs (1.1\%) with a length longer than 3000 bp (Fig. 1a). The 47,899 unigenes were composed of 23,760 unigenes (49.6\%) within the length range from 201 to $500 \mathrm{bp}$, 9224 unigenes (19.3\%) within the length range from 501 to $1000 \mathrm{bp}, 6130$ unigenes (12.8\%) within the length range from 1001 to $1500 \mathrm{bp}$, 3883 unigenes (8.1\%) within the length range from 1501 to $2000 \mathrm{bp}$, and 4902 unigenes (10.2\%) with a length longer than $2000 \mathrm{bp}$ (Fig. 1b).

\section{Sequence annotation and functional classification}

The de novo assembly yielded 47,899 unigenes. Among these unigenes, 36,289 (75.8\%) showed high similarity to known proteins in the $\mathrm{Nr}$ protein database, while 23,471 unigenes $(49.0 \%)$ were homologous to proteins in the Swiss-Prot database (Additional file 2). A total of 38,931 unigenes were annotated in the known databases.

According to the E-value distribution of the unigene hits in the $\mathrm{Nr}$ database, 24,860 (68.5\%) of the unigenes had a similarity greater than $80 \%$ to known plant sequences; 26,647 (73.4\%) of the mapped unigenes had high homology (E-value, 1.0E-30) with the available plant sequences; 22,778 (62.8\%) of the unigenes had Evalues less than $1.0 \mathrm{E}-45$; and $26.6 \%$ of the homologous sequences had E-values within the range from 1.0E-5 to $1.0 \mathrm{E}-30$ (Fig. 2a). In the sequence similarity distribution analysis, 851 (2.3\%), 2041 (5.6\%), 8537 (23.5\%), 20,953 (57.7\%), and 3907 (10.8\%) sequences were $19-40 \%$, 


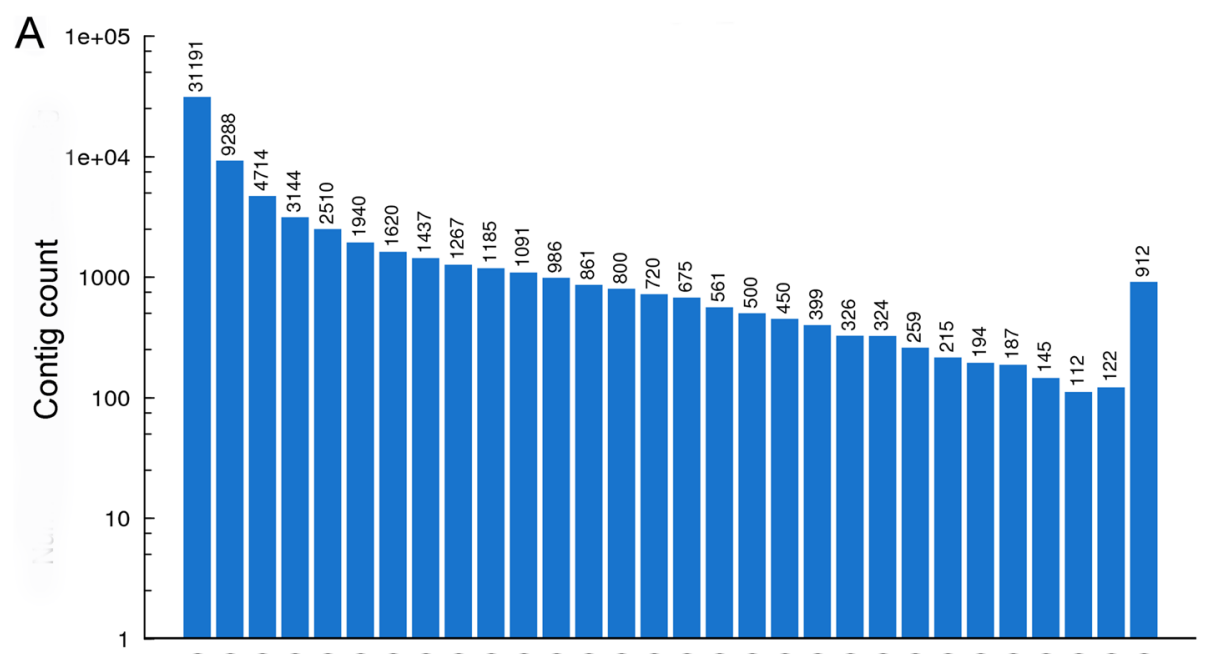

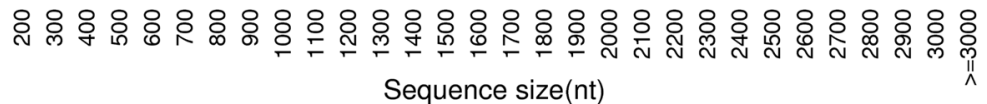

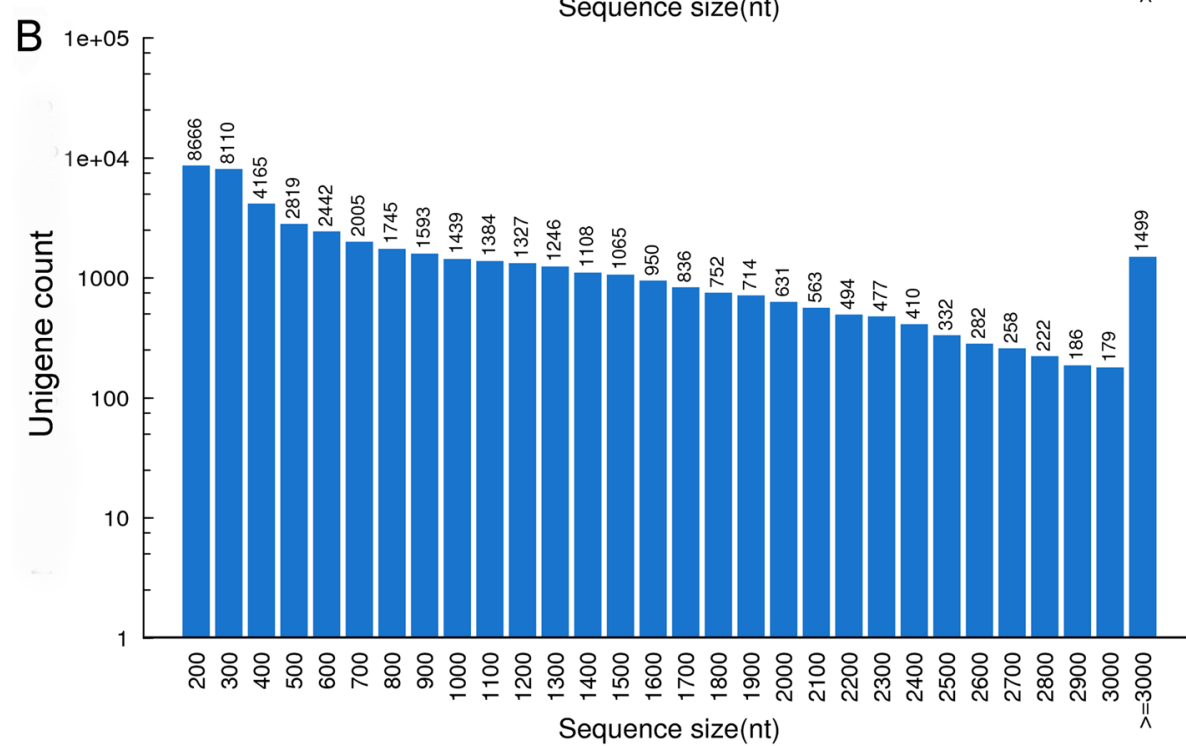

Fig. 1 Sequence length distribution of the assembled contigs (a) and unigenes (b)

$41-60 \%, 61-80 \%, 81-95 \%$, and $96-100 \%$ similar to those in the $\mathrm{Nr}$ database, respectively (Fig. 2b). For the species distribution in Leguminosae, Glycine max was ranked first with 31,682 (87.3\%) of the top BLASTx hits, followed by Medicago truncatula, Phaseolus vulgaris, Vigna unguiculata, Vigna radiata, Vigna angularis, Phaseolus aureus and Pisum sativum with 1554 (4.3\%), 497 (1.4\%), $258(0.6 \%), 80(0.2 \%), 44(0.1 \%), 26(0.1 \%)$, and $20(0.1 \%)$ hits, respectively.

In addition, $1737(4.8 \%)$ of the sequences matched sequences from other species, including 385 (1.1\%) of the sequences in Vitis vinifera (Vitaceae), 322 (0.9\%) of the sequences in Lotus corniculatus var. japonicas (Gramineae), 287 (0.8\%) of the sequences in Amygdalus persica (Rosaceae), and $71(0.2 \%)$ of the sequences in Arabidopsis thaliana (Brassicaceae) (Fig. 2c).
The unigenes with GO annotation were classified into three categories: biological processes, cellular components, and molecular functions (Fig. 3a). Among them, unigenes for biological processes accounted for the majority of the unigenes $(113,804,49.4 \%)$, followed by unigenes for cellular components $(81,937,35.6 \%)$ and unigenes for molecular function $(34,482,15.0 \%)$, covering a comprehensive range of GO categories. Among the biological process categories, unigenes of the cellular $(17,837$, $15.7 \%)$, metabolic $(17,625,15.5 \%)$, and single-organism $(12,493,11.0 \%)$ processes accounted for the majority of the unigenes. However, only a few unigenes were assigned to the categories of locomotion (25) and cell killing (2). In the category of cellular components, unigenes of cell $(20,309,34.8 \%)$ and organelle $(15,969,19.5 \%)$ components were the dominant unigenes, whereas only a few unigenes 


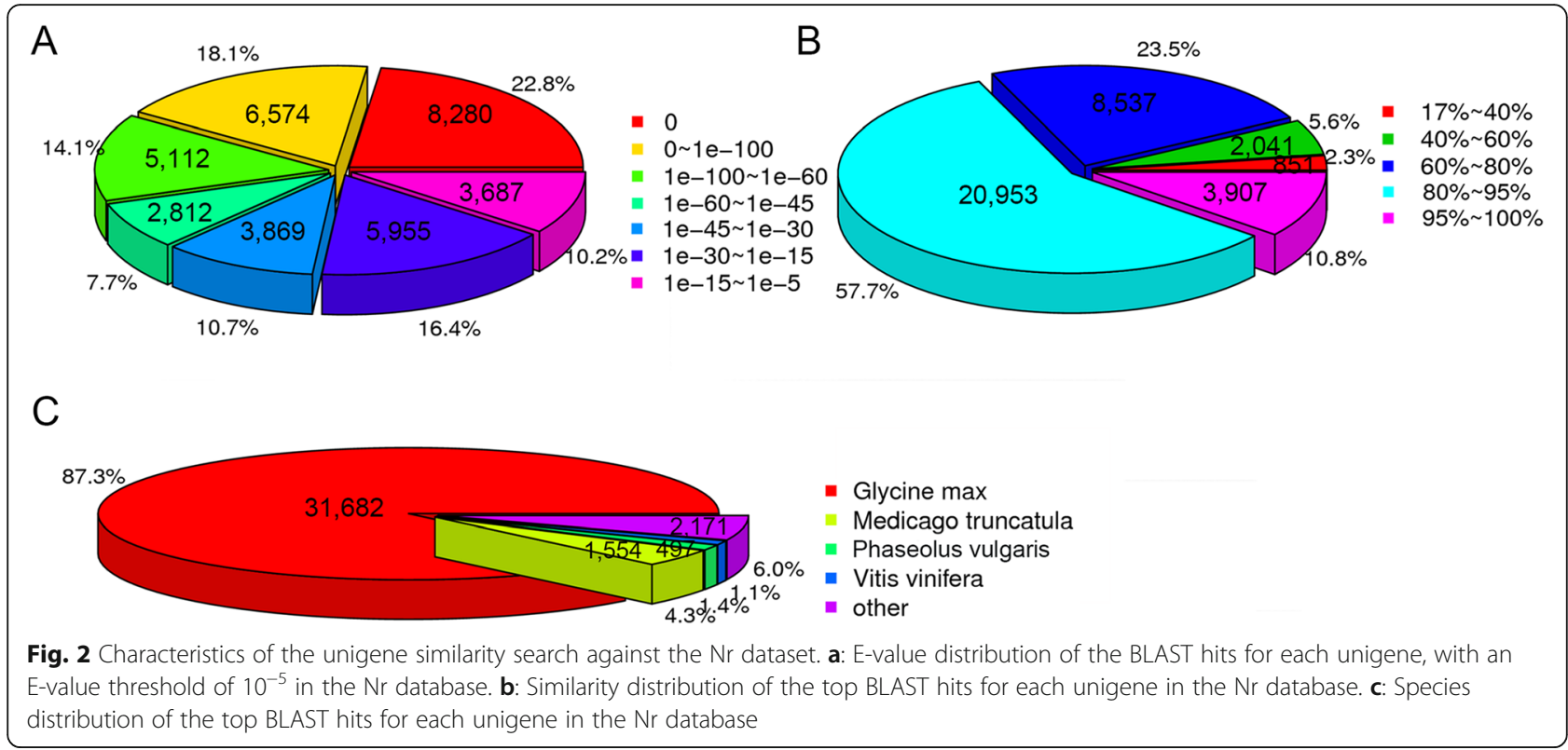

were involved in the extracellular matrix (17) and virion (7) components. In the molecular function category, catalytic activity $(14,532,42.1 \%)$ and binding $(14,479,42.0 \%)$ were the two main categories. Moreover, 2066 unigenes were assigned to transporter activity, whereas only a few unigenes were involved in translation regulator (3), protein tag (2), and channel regulator (1) activity.

According to Cluster of Orthologous Groups of proteins (COG) functional classification, 26,170 (30.8\%) unigenes were aligned to the COG database and categorized into 25 functional categories (Fig. 3b); among these categories, the general function group represented the largest group (4358 genes, 16.7\%), followed by the transcription (2683 genes, 10.3\%); replication, recombination, and repair (2158 genes, 8.3\%); signal transduction mechanism (1945 genes, 7.4\%); and posttranslational modification, chaperones, and protein turnover (1850 genes, $7.1 \%)$ groups. Nuclear structures (13 genes) and extracellular structures (5 genes) represented the smallest groups predicted by COG.

The pathway annotations of the unigene sequences were analysed using the KEGG mapper. A total of 20,654 unigenes were assigned to 5 major categories in 128 pathways that showed high similarity (Fig. 3c). Among these unigenes, metabolic pathways contained 5040 (24.4\%) unigenes, followed by biosynthesis of secondary metabolites (2390, 11.6\%), plant hormone signal transduction (1262, 6.1\%), plant-pathogen interaction (1179, 5.7\%), and endocytosis $(765,3.7 \%)$.

\section{Frequency and distribution of genic SSRs}

Among the 47,899 unigenes found in this study, 4729 (9.9\%) unigenes contained one or more SSR sequences.
Additionally, 690 (1.4\%) unigenes contained at least two independent SSR sequences, and $235(0.5 \%)$ contained compound SSRs of different motifs. These compound SSRs were not evenly distributed in various genic SSR units or groups. Tri-nucleotide (2500), di-nucleotide (1540), mono-nucleotide (1164), hexa-nucleotide (155), penta-nucleotide (137), and tetra- nucleotide (64) motifs accounted for $45.0 \%, 27.7 \%, 20.9 \%, 2.8 \%, 2.5 \%$, and $1.2 \%$, respectively; the tri-nucleotide motifs $(2500,45.0 \%)$ were the most abundant, followed by the di-nucleotide motifs (1540, 27.7\%) (Table 1).

The number of SSR repeats per locus ranged from 4 to 24, and SSRs with five repeats were the most abundant, followed by SSRs with six, seven, and twelve random repeats. Motifs with more than 17 repeats accounted for only $6.5 \%$ of the total. The $(\mathrm{A} / \mathrm{T})_{\mathrm{n}}$ repeats were the most abundant $(99.7 \%$ in mononucleotide motif) of the nucleotide repeats. The other six main motif types included the $(\mathrm{AG} / \mathrm{CT})_{\mathrm{n}}$ dinucleotide repeat $(71.2 \%)$, (AAG/CTT) $)_{\mathrm{n}}$ tri-nucleotide repeat $(31.1 \%)$, (AAGAG/CTCTT) $)_{n}$ penta-nucleotide repeat $(27.7 \%)$, (AAAG/CTTT) $)_{\mathrm{n}}$ tetra-nucleotide repeat $(23.4 \%)$, and (AAACCC/GGGTTT) ${ }_{\mathrm{n}}$ hexa-nucleotide repeat $(4.5 \%)$.

\section{Development of genic SSR markers}

A total of 5560 genic SSR markers were obtained from the 4729 SSR-containing sequences (Additional file 3). To validate their polymorphisms for 32 cowpea accessions, one set of 500 markers was randomly chosen from the above list of loci (Additional file 4). Among the tested markers, 235 primer pairs (47.0\%) produced clear amplicons with the expected sizes in nearly all of the 32 


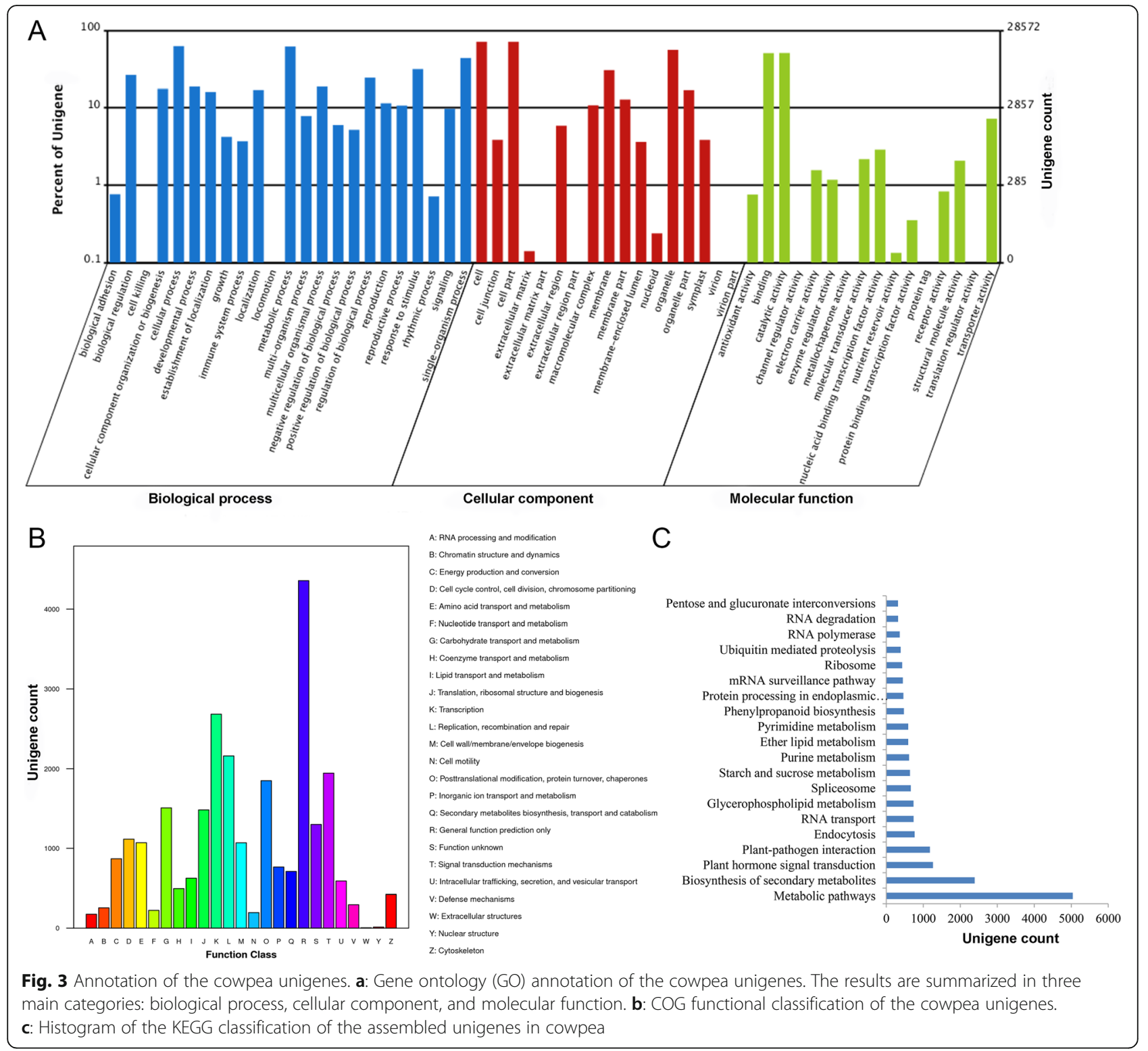

cowpea accessions, 73 primer pairs produced non-specific products, and 192 primer pairs produced no clear PCR bands. Among the successful markers, 54 (10.8\%) polymorphic genic SSR markers were identified, containing 7 di-, 29 tri-, 2 tetra-, 3 penta- and 13 hexa-motifs, while the other 181 primer pairs were monomorphic.

\section{Validation of genic SSR markers}

To confirm the 54 polymorphic genic SSRs developed in this study, we performed an extra validation in 32 diverse cowpea accessions (Additional file 5). The electrophoretic profiles of 32 cowpea accessions using marker Vu6289 are shown in Fig. 4. A total of 154 alleles were detected and scored (Table 2). The observed heterozygosity $(\mathrm{Ho})$ varied from 0.1316 (Unigene 6390) to 0.9345 (Unigene 6224), and the average was 0.5474 . The polymorphic information content (PIC) values ranged from 0.0624 (Unigene 6224) to 1.6215 (Unigene 6857), and the average was 0.8392 .

These unigene sequences of 54 polymorphic genic SSRs were subjected to BLASTn analysis, and all the sequences were similar to protein-encoding genes from mung bean (Vigna radiata) and adzuki bean (Vigna angularis). A few examples were genes for the auxinresponsive protein (IAA16), E3 ubiquitin protein ligase (DRIP2), ABC transporter, phosphomethylethanolamine $\mathrm{N}$-methyltransferase, leucine zipper protein (ATHB-14), dof zinc finger protein (DOF1.8), ethylene-responsive transcription factor (TINY) and glycine-rich cell wall structural protein (Additional file 6). 
Table 1 Summary of the numbers of repeat units in cowpea genic SSR loci

\begin{tabular}{|c|c|c|c|c|c|c|c|c|c|}
\hline \multirow[t]{2}{*}{ Repeat motif } & \multicolumn{9}{|c|}{ No. of repeats } \\
\hline & 4 & 5 & 6 & 7 & 8 & 9 & 10 & $>10$ & Total \\
\hline \multicolumn{10}{|c|}{ Mono-nucleotide (1164) } \\
\hline $\mathrm{A} / \mathrm{T}$ & - & - & - & - & - & - & - & 1161 & 1161 \\
\hline$C / G$ & - & - & - & - & - & - & - & 3 & 3 \\
\hline \multicolumn{10}{|l|}{ Di-nucleotide (1540) } \\
\hline $\mathrm{AG} / \mathrm{CT}$ & - & - & 418 & 198 & 150 & 125 & 134 & 71 & 1096 \\
\hline AT/AT & - & - & 91 & 51 & 41 & 12 & 22 & 28 & 245 \\
\hline $\mathrm{AC} / \mathrm{GT}$ & - & - & 96 & 47 & 28 & 13 & 8 & 7 & 199 \\
\hline Others & - & - & - & - & - & - & - & - & - \\
\hline \multicolumn{10}{|l|}{ Tri-nucleotide (2500) } \\
\hline $\mathrm{AAG} / \mathrm{CTT}$ & - & 397 & 237 & 137 & 6 & - & - & - & 777 \\
\hline ATC/ATG & - & 253 & 102 & 79 & 7 & - & - & - & 441 \\
\hline ACC/GGT & - & 174 & 65 & 32 & 5 & - & - & - & 276 \\
\hline AGC/CTG & - & 156 & 43 & 29 & 7 & - & - & - & 235 \\
\hline $\mathrm{AAC} / \mathrm{GTT}$ & - & 142 & 61 & 20 & 3 & - & - & - & 226 \\
\hline Others & - & 337 & 133 & 54 & 21 & - & - & - & 545 \\
\hline \multicolumn{10}{|l|}{ Tetra-nucleotide (64) } \\
\hline AAAG/CTTT & - & 13 & 2 & - & - & - & - & - & 15 \\
\hline ACTC/AGTG & - & 7 & 7 & - & - & - & - & - & 14 \\
\hline AAAT/ATTT & - & 10 & - & - & - & - & - & - & 10 \\
\hline AATG/ATTC & - & 8 & 1 & - & - & - & - & - & 9 \\
\hline Others & - & 12 & 4 & - & - & - & - & - & 16 \\
\hline \multicolumn{10}{|l|}{ Penta-nucleotide (137) } \\
\hline AAGAG/CTCTT & 38 & - & - & - & - & - & - & - & 38 \\
\hline AAAAT/ATTTT & 13 & - & - & - & - & - & - & - & 13 \\
\hline AAAAG/CTTTT & 10 & 1 & - & - & - & - & - & - & 11 \\
\hline AACAC/GTGTT & 9 & - & - & - & - & - & - & - & 9 \\
\hline Others & 62 & 4 & - & - & - & - & - & - & 66 \\
\hline \multicolumn{10}{|l|}{ Hexa-nucleotide (155) } \\
\hline AAACCC/GGGTTT & 7 & - & - & - & - & - & - & - & 7 \\
\hline AATGGC/ATTGCC & 7 & - & - & - & - & - & - & - & 7 \\
\hline AAAGAG/CTCTTT & 6 & - & - & - & - & - & - & - & 6 \\
\hline AACTTG/AAGTTC & 5 & - & - & - & - & - & - & - & 5 \\
\hline ACAGCC/CTGTGG & 5 & - & - & - & - & - & - & - & 5 \\
\hline Others & 125 & - & - & - & - & - & - & - & 125 \\
\hline Total & 287 & 1514 & 1260 & 647 & 268 & 150 & 164 & 1270 & 5560 \\
\hline$\%$ & 5.2 & 27.2 & 22.7 & 11.6 & 4.8 & 2.7 & 3.0 & 22.8 & \\
\hline
\end{tabular}

\section{Discussion}

Our transcriptomic analysis revealed a comprehensive transcriptome of the cowpea using Illumina sequencing technology, contributed a new and significant set of 54 million high-quality cDNA sequence reads, and provided a set of 47,899 unigenes for cowpea. Our analysis also identified 5560 genic SSRs; this is the first large-scale development of SSR markers in cowpea. These findings not only provide valuable information for genetic diversity, linkage mapping, gene/ QTL mapping, and marker-assisted selection in cowpea but also show promise in the search for informative molecular markers in a crop plant with few genomic resources. 


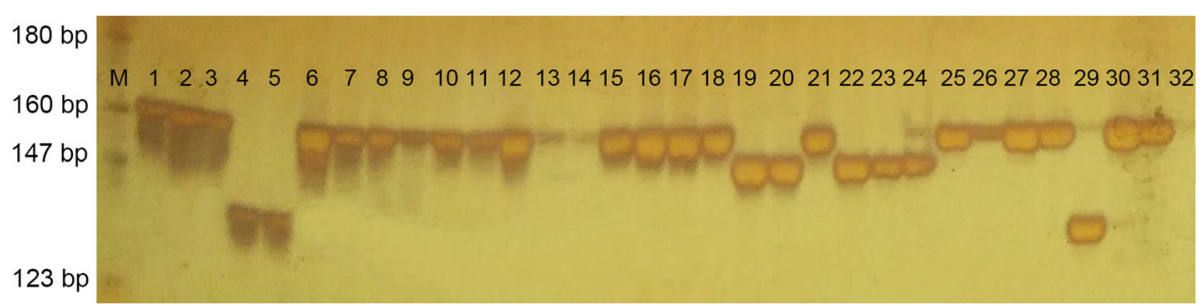

Fig. 4 An illustration of a PAGE gel showing allelic variation for the genic SSR marker Vu6289 among 32 cowpea accessions. The 32 cowpea germplasm accessions are listed in Additional file 5. M: pBR322 DNA/Mspl DNA ladder

Compared with genomic SSRs, genic SSRs, being parts of genes, are more useful as molecular markers, as they represent variation in the expressed part of the genome. Additionally, genic SSR-flanking sequences are highly conserved and show high transferability to related species or genera owing to the higher conservation of expressed sequences across species. Genic SSR markers developed in one species could be used in related species or genera for which sufficient sequence information is not available for marker development [32]. Previously, genome conservation among different legume genera was detected with DNA markers [19, 33-36]. Cowpea showed the greatest similarity with common bean, mung bean, adzuki bean, rice bean and black gram. High degrees of conservation and synteny among these five legumes were revealed through comparative mapping $[37,38]$. This may reflect the genetic relatedness and higher gene homology among these five genomes, as all are self-pollinated diploid legumes $(2 \mathrm{n}=2 \times=22)$ with a similar genome size and belong to the genus Vigna.

Genic SSRs have also been applied to analyse genetic diversity, to map QTLs controlling agronomically important traits and to increase the efficiency of markerassisted selection [32]. However, traditional Sanger sequencing of cDNAs could not provide enough unigenes or sufficient contig lengths because of the limitation of the sequenced length even if full-length cDNA libraries were adopted [39]. Transcriptomic data from RNA-Seq are useful for analysing transcriptome sequencing and assembling in many plants. This technique is rapid and cost-effective for developing microsatellite markers [21, 25] and specifically is a promising method for marker analysis in species without sequenced genomes. Our analysis has shown the usefulness of transcriptome sequencing in unigene assembly and marker development in cowpea.

In the present study, a total of 54.42 million clean reads with a length of 4,897,536,660 bp were obtained using Illumina paired-end sequencing technology. Additionally, $98.45 \%$ of the clean reads had Phred quality scores at the Q20 level, and the percentage of ambiguous " $\mathrm{N}$ " bases was $0.01 \%$, ensuring the quality of the sequencing. Furthermore, 47,899 unigenes were assembled from the cowpea transcriptome, with an average unigene length of $871 \mathrm{bp}$, which is similar to other Vigna plants, as reported in mung bean (874 bp) [40]. The average unigene length was longer than that of black gram (443 bp) [41] but was shorter than that reported in other Vigna plants such as rice bean (986 bp) [25] and adzuki bean (1213 bp) [26]. This difference may reflect a difference of species and of the assembler and parameters; for example, a longer mean length of unigenes in adzuki bean is due to the well-assembled reference genome of adzuki bean.

As a preliminary study, a total of 1071 SSRs in 15,740 cowpea unigene sequences were identified; as a result, 102 SSR markers and 1536 SNP markers were developed [20]. Genic SSRs have been broadly developed in many legume species, including common bean [21], chickpea [22], pigeonpea [23], rice bean [25], adzuki bean [26], mung bean [40], and black gram [41]. A total of 3011, $7947,13,134$ and 1840 genic SSRs were identified from 71,929 rice bean unigenes [24], 65,950 adzuki bean unigenes [25], 48,693 mung bean unigenes [40] and 48,291 black gram transcript contigs [41], respectively. In this study, a total of 5560 genic SSR markers were developed from 4729 SSR-containing unigene sequences. To the best of our knowledge, this is the first large-scale development of SSR markers in cowpea. The new SSR sequences and genic SSR markers provide not only important genomic resources for basic research but also some new opportunities to find closely linked markers for traits of agronomic importance for the genetic improvement of cowpea.

To determine the polymorphism levels of the developed genic SSR markers, we evaluated 500 genic SSR loci, and 235 primer pairs (47.0\%) produced successful amplicons. Additionally, 54 new polymorphic genic SSR markers were characterized and validated. A low polymorphism level was detected in 32 cowpea accessions, and the valid primer ratio was only $10.8 \%$ (54 in 500), which is lower than that from previous reports in other related legume species, including mung bean (33\%) [40], black gram (58.2\%) [41], common bean (71.3\%) [42] and chickpea (47.3\%) [43] but higher than that reported in rice bean (7.6\%) [25] and adzuki bean (7.6\%) [26]. The 
Table 2 Characteristics of the 54 polymorphic SSR markers validated in 32 different cowpea accessions

\begin{tabular}{|c|c|c|c|}
\hline Gene locus & $\mathrm{Na}$ & Ho & PIC \\
\hline Unigene4200 & 2 & 0.1316 & 0.4113 \\
\hline CL2002Contig1 & 2 & 0.5192 & 0.3457 \\
\hline Unigene4708 & 2 & 0.5023 & 0.0688 \\
\hline CL4898Contig2 & 2 & 0.7506 & 0.3648 \\
\hline CL4580Contig2 & 2 & 0.4918 & 0.4188 \\
\hline CL150Contig2 & 2 & 0.8169 & 0.3648 \\
\hline Unigene4211 & 3 & 0.4289 & 0.2149 \\
\hline Unigene16847 & 3 & 0.4644 & 0.2688 \\
\hline CL275Contig1 & 3 & 0.5277 & 0.2205 \\
\hline Unigene11213 & 3 & 0.4604 & 0.3648 \\
\hline Unigene11595 & 2 & 0.6362 & 0.2392 \\
\hline Unigene10812 & 2 & 0.9273 & 0.3236 \\
\hline CL2728Contig2 & 3 & 0.4364 & 0.2688 \\
\hline Unigene13454 & 2 & 0.5119 & 0.1638 \\
\hline Unigene5616 & 2 & 0.5119 & 0.3285 \\
\hline Unigene8092 & 2 & 0.5480 & 0.3739 \\
\hline Unigene3540 & 2 & 0.5783 & 0.2938 \\
\hline CL2572Contig1 & 2 & 0.5006 & 0.3833 \\
\hline Unigene9045 & 2 & 0.6746 & 0.3690 \\
\hline Unigene15775 & 2 & 0.6746 & 0.4605 \\
\hline Unigene5773 & 2 & 0.7175 & 0.1638 \\
\hline Unigene15736 & 2 & 0.5119 & 0.3610 \\
\hline CL2115Contig1 & 3 & 0.4797 & 0.3747 \\
\hline Unigene17353 & 2 & 0.8169 & 0.4634 \\
\hline Unigene10711 & 2 & 0.4938 & 0.3705 \\
\hline Unigene14297 & 2 & 0.5692 & 0.4217 \\
\hline CL2511Contig5 & 2 & 0.7428 & 0.3318 \\
\hline Unigene6203 & 3 & 0.3682 & 0.5556 \\
\hline Unigene6211 & 3 & 0.4156 & 0.5193 \\
\hline Unigene6270 & 3 & 0.4328 & 0.4956 \\
\hline Unigene6222 & 2 & 0.7013 & 0.2503 \\
\hline Unigene6346 & 4 & 0.4311 & 0.4631 \\
\hline Unigene6311 & 2 & 0.5402 & 0.3498 \\
\hline Unigene6298 & 3 & 0.5684 & 0.3833 \\
\hline Unigene6357 & 6 & 0.1972 & 0.7588 \\
\hline Unigene6375 & 2 & 0.7096 & 0.2447 \\
\hline Unigene6277 & 6 & 0.3533 & 0.5969 \\
\hline Unigene6300 & 2 & 0.8734 & 0.1167 \\
\hline Unigene6258 & 4 & 0.4789 & 0.4777 \\
\hline Unigene6224 & 2 & 0.9345 & 0.0624 \\
\hline Unigene6249 & 3 & 0.8064 & 0.1807 \\
\hline Unigene6195 & 4 & 0.6277 & 0.1787 \\
\hline Unigene6192 & 4 & 0.4153 & 0.3410 \\
\hline
\end{tabular}

Table 2 Characteristics of the 54 polymorphic SSR markers validated in 32 different cowpea accessions (Continued)

\begin{tabular}{llll}
\hline Unigene6218 & 2 & 0.8169 & 0.4641 \\
Unigene6327 & 3 & 0.4645 & 0.1638 \\
Unigene6333 & 2 & 0.8169 & 0.1545 \\
Unigene6683 & 7 & 0.1949 & 0.4690 \\
Unigene6214 & 2 & 0.6746 & 0.1638 \\
Unigene6265 & 5 & 0.2329 & 0.7621 \\
Unigene6390 & 10 & 0.1316 & 0.2688 \\
Unigene6770 & 3 & 0.4492 & 0.7172 \\
Unigene6857 & 2 & 0.4973 & 0.8392 \\
Unigene6347 & 3 & 0.3254 & 0.4598 \\
Unigene6213 & 2 & 0.3124 & 0.3153 \\
Mean & 2.8 & 0.5474 & 0.3440 \\
St. & 1.5 & 0.1926 & 0.1476 \\
\hline $\begin{array}{l}\text { Number of alleles }(\mathrm{Na}) \text {, observed heterozygosity } \\
\text { information content }(P I C)\end{array}$ & &
\end{tabular}

ratio of effective to non-effective primer design in these results was higher than that reported from BAC-derived SSRs in common bean [44] or from the screening of non-enriched small-insert genomic libraries [45]. The location of primers across splice sites or regions of poor sequence quality could explain the non-amplification. More than two-thirds of the genic SSR markers produced successful amplicons, suggesting that the quality of our assembled unigenes is high.

In this study, the tri-nucleotide motifs (2500, 20.9\%) and di-nucleotide motifs (1540, 27.7\%) were the most abundant. The types of motifs found in SSR loci were similar to the previous results on microsatellites in plants [46]. A relatively large proportion of the di- and tri-nucleotide repeats in EST sequences has been reported in common bean [21] and chickpea [22]. The most common tri-nucleotide repeat found in cowpea was AAG/CTT, followed by ACC/GGT and AAC/GTT. The most common di-nucleotide repeat was AG/CT, followed by AT/AT and AC/GT. The results are similar to previous studies in adzuki bean [47], common bean [48], and fava bean [49]. Additionally, the number of alleles in most of the successfully amplified SSRs was less than four. The PIC values in the current study ranged from 0.0624 to 1.6215 , with an average of 0.8392 , which is in line with previous reports for cowpea [20] and mung bean [40] SSRs.

Applicable microsatellite loci were identified, and some of them may be useful in genetic research. However, there are some limitations in our study. The cDNA library was established using only one cowpea variety, which may cause an ascertainment bias. Second, only 500 of 5560 SSR markers were randomly selected for further validation, limiting their application potential. 
Third, more efficient RNA-Seq designs are needed to enhance marker development in transcriptomic studies. Fourth, the polymorphism rate is relatively low. Such a low rate of polymorphisms may be due to (i) a large intron between primers that would prevent amplification of the genomic sequence based on the transcribed mRNA sequence, (ii) the priming sites being disrupted by unrecognized intron splice sites, and (iii) low-quality sequences, fragmented assembly or assembly errors. The failure of 24 primer pairs to generate amplicons might be caused by long intervening introns, which would prevent PCR amplification of selected markers.

\section{Conclusions}

The transcriptomic analysis in our study was fruitful, providing not only a valuable set of genomic data characterizing genes for important agronomic traits in Vigna unguiculata but also a new set of genic SSR markers for further genetic studies and breeding in cowpea and related Vigna species. Our analysis also showed the promise of using the RNA-Seq technique for SSR development in crop plants, such as cowpea, with few or no genomic resources.

\section{Methods}

\section{Plant materials}

A set of 32 Chinese cowpea accessions was used in SSR marker testing, which included 14 accessions from Hunan province, 9 accessions from Hubei province, and 9 accessions from Anhui province. These cowpea germplasm accessions were obtained from the National Gene Bank (China, Beijing). The cowpea seedlings were grown in plastic pots $(20 \mathrm{~cm} \times 15 \mathrm{~cm} \times 15 \mathrm{~cm})$ under a $14 / 10 \mathrm{~h}$ photoperiod at $25{ }^{\circ} \mathrm{C}$ (day) and $20^{\circ} \mathrm{C}$ (night) in a greenhouse at the Institute of Crop Science, Chinese Academy of Agricultural Sciences (China, Beijing, $116^{\circ}$ $\left.46^{\prime} \mathrm{E}, 39^{\circ} 92^{\prime} \mathrm{N}\right)$. At the three-week-old seedling stage, five plants of "Zhongjiang No. 1" were transferred to a programmable incubator for dehydration for $3 \mathrm{~h}$ at $28{ }^{\circ} \mathrm{C}$, termed drought stress, and the seedlings were then harvested, frozen immediately in liquid nitrogen, and stored at $-80{ }^{\circ} \mathrm{C}$ for RNA-Seq.

\section{RNA extraction}

Total RNA was isolated from three different tissues, including roots, stems, and leaves, of five cowpea seedlings, and these RNA samples were mixed according to the equivalent concentration of these RNA samples were mixed, and $20 \mu \mathrm{g}$ of RNA was quickly frozen in liquid nitrogen and then stored at $-80{ }^{\circ} \mathrm{C}$. The RNA was isolated with TRIZOL reagent (Invitrogen, Carlsbad, CA, USA) following the manufacturer's instructions. The residual DNA was removed by incubation with RNasefree DNase I (TaKaRa, Kyoto, Japan) for $30 \mathrm{~min}$ at $37{ }^{\circ} \mathrm{C}$.
The concentration and integrity of the total RNA was measured by a 2100 Bioanalyzer (Agilent, Santa Clara, CA) at $260 \mathrm{~nm}$ and $280 \mathrm{~nm}$ and checked for degradation by RNase-free agarose gel electrophoresis. The RNA was further quantified with an RNA Nanochip. More than $20 \mu \mathrm{g}$ of total RNA (concentration $\geq 500 \mathrm{ng} / \mu \mathrm{L}$, OD260/ $280=1.8-2.2$, OD260/230 $\geq 2.0$, and RNA integrity number $(\mathrm{RIN}) \geq 8.0$ ) was used to create the full-length cDNA library.

\section{cDNA library establishment and sequencing}

The cDNA library was constructed using the NEBNext Ultra RNA Library Prep Kit (NEB, Ipswich, USA) according to the manufacturer's instructions [50]. The total RNA was used to isolate poly(A) RNA using poly$\mathrm{T}$ oligo-attached magnetic beads (Illumina, San Diego, CA, USA). The poly(A) RNA was then fragmented into smaller pieces using divalent cations at $94{ }^{\circ} \mathrm{C}$ for $4 \mathrm{~min}$. First-strand cDNA was synthesized using SuperScript II reverse transcriptase and random primers. Secondstrand cDNA was synthesized by adding GEX second strand buffer, dNTPs, RNase $\mathrm{H}$, and DNA polymerase I. The cDNA fragments were further subjected to end repair and phosphorylation with $\mathrm{T}_{4}$ DNA polymerase, Klenow DNA polymerase, and $\mathrm{T}_{4}$ polynucleotide kinase. The repaired cDNA fragments were then 3 '-end adenylated with Klenow Exo and ligated with PE adapters. The products of this ligation reaction were purified through $2 \%(w / v)$ TAE-agarose gel, and templates of different sizes were selected for downstream enrichment. The cDNA fragments $(200 \pm 25 \mathrm{bp})$ were finally excised from the gel and amplified via PCR to enrich the cDNA template. Purified cDNA templates were enriched by 15 cycles of PCR amplification using PE1.0 and PE2.0 primers and Phusion DNA Polymerase (NEB, USA). The cDNA library was constructed and sequenced in one lane on the Illumina Hiseq 2500 platform (Novogene, Beijing, China) with Illumina paired-end sequencing technology, and the mean fragment length was $200 \mathrm{bp}( \pm 25 \mathrm{bp}$ ) [51]. Finally, the raw data for all the reads were collected from the Illumina system. The raw sequences were submitted to the sequence read archive (SRA) at the National Center for Biotechnology Information (NCBI) under the submission IDs SRR4897234 and SRX2323067 and study accession SRP092517.

\section{Data filtering and de novo assembly}

Prior to transcriptome assembly, a stringent filtering process was carried out on the raw sequencing reads. Raw reads in fastq format were initially processed using in-house Perl scripts [51]. All reads with more than 10\% of bases having a poor quality score $(\mathrm{Q}<20)$ or noncoding RNA, and ambiguous sequences containing an 
excess of $\mathrm{N}$ nucleotides, were removed by Seqclean. The reads were preprocessed to eliminate low-quality reads (reads with ambiguous " $\mathrm{N}$ " bases $>5 \%$ and more than $10 \%$ $\mathrm{Q}<20$ bases). Reads were discarded that failed to pass the failed-chastity filter based on the relation "failed-chastity $\leq 1$ ". In the first 25 cycles, the chastity threshold was less than 0.6 for filtering. In this step, clean data were obtained by removal of adapter-containing, poly- $\mathrm{N}$-containing and low-quality reads from the raw data. Meanwhile, Q20, Q30, and the GC content of the clean data were calculated. All downstream analyses were based on the highquality clean data. A Perl pipeline was used for analysing sequence data, and de novo transcriptome assembly was performed using the program Trinity [52]. The Trinity assembler was used with the inchworm k-mer method, and all the server resources were set to unlimited. The sequences without redundancy, containing the fewest Ns and not being extended on either end, could be defined as unigenes.

\section{Unigene annotation and classification}

The annotation of unigenes was carried out with various bioinformatics procedures. First, these unigenes were used to search various protein databases including $\mathrm{Nr}$, Swiss-Prot, COG, and KEGG (E-value $\leq 1.0 \mathrm{E}-5$ ), retrieving proteins with the highest sequence similarity annotated to each unigene. Second, with nucleotide-based annotation, Blast2GO was used to obtain GO annotation categories according to molecular function, biological process and cellular component ontologies [53]. Third, ESTs were annotated into KEGG pathways with the KEGG Automatic Annotation Server (KAAS) [54] using the single-directional best hit (SBH) method. The unigene sequences were also aligned to the COG database to classify and predict their possible functions.

\section{SSR search and primer design}

To amplify a sequence with appropriate length ( $>100 \mathrm{bp})$ based on ensuring the quality of the primer, only sequences for which both ends of the SSR had lengths greater than $150 \mathrm{bp}$ were used to design these primers. In this study, the minimum number of repeats for different SSR markers was chosen as follows: eleven for mononucleotide, six for di-nucleotide, five for tri- and tetranucleotide, and four for penta- and hexa-nucleotide motifs. Primers were designed based on the flanking sequences of these SSRs using Primer Premier 3.0 (http://sourceforge.net/projects/primer3) under the following criteria: primer length 18-22 bp (optimally $20 \mathrm{bp}$ ), $\mathrm{Tm}$ of $50-60{ }^{\circ} \mathrm{C}$ (no more than a $4{ }^{\circ} \mathrm{C}$ difference between the annealing temperatures of the forward and reverse primers), and PCR product length in the range of 100-300 bp. Primers were synthesized by Qingke Biotech (Beijing, China).

\section{Genic SSR characterization and validation}

The identified SSR markers were amplified and validated with 32 accessions of cowpea, including fourteen accessions from Hunan Province, nine accessions from Hubei Province and nine accessions from Anhui Province (Additional file 5). These cowpea accessions were preserved in the National Gene Bank at the Institute of Crop Science, Chinese Academy of Agricultural Sciences (Beijing, China, 116 $46^{\prime} \mathrm{E}, 39^{\circ} 92^{\prime} \mathrm{N}$ ). Genomic DNA was extracted from young leaves of five plants for each accession using the cetyl trimethyl ammonium bromide (CTAB) method [23]. DNA quality and quantity were analysed by $1 \%$ agarose gel electrophoresis. PCR was performed in a system of $20 \mu \mathrm{L}$ containing $0.5 \mathrm{U}$ of Taq polymerase, $1 \times$ PCR Buffer, $1.5 \mathrm{mM}$ $\mathrm{MgCl}_{2}, 25 \mu \mathrm{M}$ dNTP (Qingke Biotech, Beijing, China), $0.4 \mu \mathrm{M}$ of each primer, and $30 \mathrm{ng}$ of genomic DNA. PCR was carried out on a Bio-Rad $\mathrm{T}_{100}{ }^{\mathrm{m}}$ Thermal Cycler (Bio-Rad, USA). PCR amplification was performed as follows: $94{ }^{\circ} \mathrm{C}$ for $4 \mathrm{~min}$ and 30 cycles of $94{ }^{\circ} \mathrm{C}$ for $30 \mathrm{~s}, 55{ }^{\circ} \mathrm{C}$ for $30 \mathrm{~s}$ and $72{ }^{\circ} \mathrm{C}$ for $30 \mathrm{~s}$. The final extension was carried out at $72{ }^{\circ} \mathrm{C}$ for $5 \mathrm{~min}$. The PCR products obtained were verified in $6.0 \%$ non-denaturing PAGE (polyacrylamide gel electrophoresis) using the silver staining method. Fragment sizes were estimated with the pBR322 DNA/MspI DNA ladder (Kangwei Biotech, Beijing, China). To determine the functions of the polymorphic genic SSRs, the unigene sequences used for the development of these markers were subjected to a BLASTn analysis against a non-redundant database of legume sequences.

\section{Additional files}

Additional file 1: Sequences of all unigenes. (XLS $4765 \mathrm{~kb}$ )

Additional file 2: Unigenes that were annotated in the $\mathrm{Nr}$ database. (XLS 7296 kb)

Additional file 3: A list of 5560 cowpea genic SSR primers developed in this study. (XLS 764 kb)

Additional file 4: Characteristics of 500 primer pairs derived from cowpea genic SSRs. (XLS 199 kb)

Additional file 5: A list of 32 cowpea germplasm accessions used in this study. (DOC $69 \mathrm{~kb}$ )

Additional file 6: The putative proteins identified by BLASTX of 54 unigene sequences containing polymorphic genic SSRs. (XLS 35 kb)

\section{Abbreviations}

EST: Expressed sequence tag; Ho: Observed heterozygosity; Na: Alleles; PIC: Polymorphic information content; SSR: Simple sequence repeat

\section{Acknowledgments}

We are grateful to Dr. M. W. Blair (Department of Agricultural and Environmental Sciences, Tennessee State University) for his valuable suggestions on the improvement of the manuscript. 


\section{Funding}

This work was funded by the Chinese Agricultural Academy of Sciences (CAAS) Agricultural Science and Technology Innovation Program (ASTIP) to $\mathrm{HC}$, and China Agriculture Research System (CARS-09).

\section{Availability of data and materials}

Raw data supporting our findings can be found at the National Center for Biotechnology Information (NCBI) with the accession number SRP092517.

\section{Authors' contributions}

$\mathrm{HC}$ and XC conceived and designed the experiments. HC performed bioinformatic analysis, and primer design. $\mathrm{XL}$ and $\mathrm{LH}$ worked for the validation identification of SSR markers. LW and SW prepared all the seeds of cowpea. HC drafted and revised the manuscript. All authors read and approved the final manuscript.

\section{Ethics approval and consent to participate}

Not applicable.

\section{Consent for publication}

Not applicable.

\section{Competing interests}

The authors declare that they have no competing interests.

\section{Publisher's Note}

Springer Nature remains neutral with regard to jurisdictional claims in published maps and institutional affiliations.

Received: 16 July 2016 Accepted: 28 June 2017

Published online: 11 July 2017

\section{References}

1. Timko MP, Rushton PJ, Laudeman TW, Bokowiec MT, Chipumuro E, Cheung $F$, et al. Sequencing and analysis of the gene-rich space of cowpea. BMC Genomics. 2008:9:103.

2. Muchero W, Diop NN, Bhat PR, Fenton RD, Wanamaker S, Pottorff M, et al. A consensus genetic map of cowpea [Vigna unguiculata $(L)$ Walp.] and synteny based on EST-derived SNPs. Proc Natl Acad Sci U S A. 2009;106(43): 18159-64.

3. Badiane FA, Gowda BS, Cisse N, Diouf D, Sadio O, Timko MP. Genetic relationship of cowpea (Vigna unguiculata) varieties from Senegal based on SSR markers. Genet Mol Res. 2012;11(1):292-304.

4. Xiong H, Shi A, Mou B, Qin J, Motes D, Lu W, et al. Genetic diversity and population structure of cowpea (Vigna unguiculata L. Walp). PLoS One. 2016;11:e0160941.

5. Diouf D. Recent advances in cowpea [Vigna unguiculata (L.) Walp.] "omics" research for genetic improvement. Afr J Biotechnol. 2011;10(15):2803-10.

6. Town CD. Annotating the genome of Medicago truncatula. Curr Opin Plant Biol. 2006;9(2):122-7.

7. Sato S, Tabata S. lotus japonicus as a platform for legume research. Curr Opin Plant Biol. 2006;9(2):128-32.

8. Sato S, Nakamura Y, Asamizu E, Isobe S, Tabata S. Genome sequencing and genome resources in model legumes. Plant Physiol. 2007;144(2):588-93.

9. Varshney RK, Chen W, Li Y, Bharti AK, Saxena RK, Schlueter JA, Donoghue MT, Azam S, Fan G, Whaley AM, et al. Draft genome sequence of pigeonpea (Cajanus cajan), an orphan legume crop of resource-poor farmers. Nat Biotechnol. 2012:30(1):83-9.

10. Varshney RK, Song C, Saxena RK, Azam S, Yu S, Sharpe AG, Cannon S, Baek J, Rosen BD, Tar'an B, et al. Draft genome sequence of chickpea (Cicer arietinum) provides a resource for trait improvement. Nat Biotechnol. 2013; 31(3):240-6.

11. Schmutz J, Mcclean PE, Mamidi S, Wu GA, Cannon SB, Grimwood J, et al, A reference genome for common bean and genome-wide analysis of dual domestications. Nat Genet. 2014:46:707-13.

12. Kang YJ, Kim SK, Kim MY, Lestari P, Kim KH, Ha BK, et al. Genome sequence of mungbean and insights into evolution within Vigna species. Nat Commun. 2014;5:5443.

13. Yang K, Tian Z, Chen C, Luo L, Zhao B, Wang Z, et al. Genome sequencing of adzuki bean (Vigna angularis) provides insight into high starch and low fat accumulation and domestication. Proc Natl Acad Sci U S A. 2015;112(43): 13213-8.

14. Menancio-Hautea D, Fatokun CA, Kumar L, Danesh D, Young ND. Comparative genome analysis of mungbean (Vigna radiata $\mathrm{L}$. Wilczek) and cowpea ( $V$. unguiculata L. Walpers) using RFLP mapping data. Theor Appl Genet. 1993:86(7):797-810.

15. Ba FS, Pasquet RS, Gepts P. Genetic diversity in cowpea [Vigna unguiculata (L.) Walp.] as revealed by RAPD markers. Genet Resour Crop Evol. 2004;51(5): 539-50.

16. Ouedraogo JT, Gowda BS, Jean M, Close TJ, Ehlers JD, Hall AE, et al. An improved genetic linkage map for cowpea (Vigna unguiculata L.) combining AFLP, RFLP, RAPD, biochemical markers, and biological resistance traits. Genome. 2002:45(1):175-88.

17. Varshney RK, Chabane K, Hendre PS, Aggarwal RK, Graner A. Comparative assessment of EST-SSR, EST-SNP and AFLP markers for evaluation of genetic diversity and conservation of genetic resources using wild, cultivated and elite barleys. Plant Sci. 2007;173(6):638-49.

18. Asare AT, Gowda BS, Galyuon IKA, Aboagye LL, Takrama JF, Timko MP Assessment of the genetic diversity in cowpea (Vigna unguiculata L. Walp.) germplasm from Ghana using simple sequence repeat markers. Plant Genet Resour. 2010:8(2):142-50.

19. Diouf D, Hilu KW. Microsatellites and RAPD markers to study genetic relationships among cowpea breeding lines and local varieties in Senegal. Genet Resour Crop Evol. 2005;52(8):1057-67.

20. Gupta SK, Gopalakrishna T. Development of unigene-derived SSR markers in cowpea (Vigna unguiculata) and their transferability to other Vigna species. Genome. 2010;53:508-23.

21. Blair MW, Hurtado N, Chavarro CM, Munoz-Torres MC, Giraldo MC, Pedraza F, et al. Gene-based SSR markers for common bean (Phaseolus vulgaris L.) derived from root and leaf tissue ESTs: an integration of the BMc series. BMC Plant Biol. 2011;11:50.

22. Choudhary S, Sethy NK, Shokeen B, Bhatia S. Development of chickpea ESTSSR markers and analysis of allelic variation across related species. Theor Appl Genet. 2009;118(3):591-608.

23. Dutta S, Kumawat G, Singh BP, Gupta DK, Singh S, Dogra V, et al. Development of genic-SSR markers by deep transcriptome sequencing in pigeonpea [Cajanus cajan (L.) Millspaugh]. BMC Plant Biol. 2011:11:17.

24. Xin D, Sun J, Wang J, Jiang H, Hu G, Liu C, Chen Q. Identification and characterization of SSRs from soybean (Glycine max) ESTs. Mol Bioly Rep. 2012;39(9):9047-57.

25. Chen H, Chen X, Tian J, Yang Y, Liu Z, Hao X, et al. Development of genebased SSR markers in rice bean (Vigna umbellata L.) based on transcriptome data. PLoS One. 2016:11(3):e0151040.

26. Chen H, Liu L, Wang L, Wang S, Somta P, Cheng X. Development and validation of EST-SSR markers from the transcriptome of adzuki bean (Vigna angularis). PLoS One. 2015;10(7):e0131939.

27. Gupta SK, Bansal R, Gopalakrishna T. Development and characterization of genic SSR markers for mungbean (Vigna radiata (L.) Wilczek). Euphytica. 2014;195(2):245-58.

28. Boukar O, Fatokun CA, Huynh BL, Roberts PA, Close TJ. Genomic tools in cowpea breeding programs: status and perspectives. Front Plant Sci. 2016;7:757.

29. Pazos-Navarro M, Dabauza M, Correal E, Hanson K, Teakle N, Real D, et al. Next generation DNA sequencing technology delivers valuable genetic markers for the genomic orphan legume species. BMC Genet. 2011;12:104.

30. Wei WL, Qi XQ, Wang LH, Zhang YX, Hua W, Li DH, et al. Characterization of the sesame (Sesamum indicum L.) global transcriptome using Illumina paired-end sequencing and development of EST-SSR markers. BMC Genomics. 2011;12:451.

31. Zhang JA, Liang S, Duan JL, Wang J, Chen SL, Cheng ZS, et al. De novo assembly and characterisation of the transcriptome during seed development, and generation of genic-SSR markers in peanut (Arachis hypogaea L.). BMC Genomics. 2012;13:90.

32. Varshney RK, Graner A, Sorrells ME. Genic microsatellite markers in plants: features and applications. Trends Biotechnol. 2005;23:48-55.

33. Verma P, Sharma TR, Srivastava PS, Abdin MZ, Bhatia S. Exploring genetic variability within lentil (Lens culinaris Medik.) and across related legumes using a newly developed set of microsatellite markers. Mol Biol Rep. 2014;41:5607-25. 
34. Chen H, Liu L, Wang L, Wang S, Wang ML, Cheng X. Development of SSR markers and assessment of genetic diversity of adzuki bean in the Chinese germplasm collection. Mol Breeding. 2015;35:191.

35. Chen H, Qiao L, Wang L, Wang S, Blair MW, Cheng X. Assessment of genetic diversity and population structure of mung bean (Vigna radiata) germplasm using EST-based and genomic SSR markers. Gene. 2015;566:175-83.

36. Wang $L$, Chen H, Bai P, Wu J, Wang S, Blair MW, et al. The transferability and polymorphism of mung bean SSR markers in rice bean germplasm. Mol Breeding. 2015;35:77.

37. Gupta SK, Gopalakrishna T. Advances in genome mapping in orphan grain legumes of genus Vigna. Indian J Genet. 2013;73(1):1-13.

38. Vasconcelos EV, Fonseca AFD, Pedrosa-Harand A, Bortoleti KCD, Benko-Iseppon $A M$, da Costa AF, et al. Intra- and interchromosomal rearrangements between cowpea [Vigna unguiculata (L.) Walp.] and common bean (Phaseolus vulgaris L.) revealed by BAC-FISH. Chromosome Res. 2015;23:253-66.

39. Kudapa H, Bharti AK, Cannon SB, Farmer AD, Mulaosmanovic B, Kramer R, et al. A comprehensive transcriptome assembly of pigeonpea (Cajanus cajan L.) using sanger and second-generation sequencing platforms. Mol Plant. 2012;5:1020-8.

40. Chen H, Wang L, Wang S, Liu C, Blair MW, Cheng X. Transcriptome sequencing of mung bean (Vigna radiata $L$.) genes and the identification of EST-SSR markers. PLoS One. 2015;10:e0120273.

41. Souframanien J, Reddy KS. De novo assembly, characterization of immature seed transcriptome and development of genic-SSR markers in black gram [Vigna mungo (L.) Hepper]. PLoS One. 2015;10:e0128748.

42. Hanai LR, de Campos T, Camargo LE, Benchimol LL, de Souza AP, Melotto $M$, et al. Development, characterization, and comparative analysis of polymorphism at common bean SSR loci isolated from genic and genomic sources. Genome. 2007;50:266-77.

43. Nayak SN, Zhu H, Varghese N, Datta S, Choi HK, Horres R, et al. Integration of novel SSR and gene-based SNP marker loci in the chickpea genetic map and establishment of new anchor points with Medicago truncatula genome. Theor Appl Genet. 2010;120:1415-41.

44. Cordoba JM, Chavarro C, Schlueter JA, Jackson SA, Blair MW. Integration of physical and genetic maps of common bean through BAC-derived microsatellite markers. BMC Genomics. 2010;11:436.

45. Blair MW, Torres MM, Pedraza F, Giraldo MC, Buendia HF, Hurtado N. Development of microsatellite markers for common bean (Phaseolus vulgaris L.) based on screening of non-enriched, small-insert genomic libraries. Genome. 2009;52(9):772-82.

46. La Rota M, Kantety RV, Yu JK, Sorrells ME. Nonrandom distribution and frequencies of genomic and EST-derived microsatellite markers in rice, wheat, and barley. BMC Genomics. 2005;6:23.

47. Chankaew S, Isemura T, Isobe S, Kaga A, Tomooka N, Somta P, et al. Detection of genome donor species of neglected tetraploid crop Vigna reflexo-pilosa (creole bean), and genetic structure of diploid species based on newly developed EST-SSR markers from adzuki bean (Vigna angularis). PLoS One. 2014;9(8):e104990.

48. Blair MW, Cordoba JM, Munoz C, Yuyo DK. BAC-end microsatellites from intra and inter-genic regions of the common bean genome and their correlation with cytogenetic features. PLoS One. 2014;9(9):e101873.

49. Gong YM, Xu SC, Mao WH, Li ZY, Hu QZ, Zhang GW. Genetic diversity analysis of faba bean (Vicia faba L.) based on EST-SSR markers. Agr Sci China. 2011;10(6):838-44.

50. Rhodes J, Beale MA, Fisher MC. Illuminating choices for library prep: a comparison of library preparation methods for whole genome sequencing of Cryptococcus neoformans using Illumina HiSeq. PLoS One. 2014;9:e113501.

51. Wang Z, Gerstein M, Snyder M. RNA-Seq: a revolutionary tool for transcriptomics. Nat Rev Genet. 2009;10:57-63.

52. Haas BJ, Papanicolaou A, Yassour M, Grabherr M, Blood PD, Bowden J, et al. De novo transcript sequence reconstruction from RNA-Seq using the Trinity platform for reference generation and analysis. Nat Protoc 2013;8(8):1494-512.

53. Conesa A, Gotz S, Garcia-Gomez JM, Terol J, Talon M, Robles M. Blast2GO: a universal tool for annotation, visualization and analysis in functional genomics research. Bioinformatics. 2005;21(18):3674-6.

54. Moriya Y, Itoh M, Okuda S, Yoshizawa AC, Kanehisa M. KAAS: an automatic genome annotation and pathway reconstruction server. Nucleic Acids Res. 2007;35:182-5

\section{Submit your next manuscript to BioMed Central and we will help you at every step:}

- We accept pre-submission inquiries

- Our selector tool helps you to find the most relevant journal

- We provide round the clock customer support

- Convenient online submission

- Thorough peer review

- Inclusion in PubMed and all major indexing services

- Maximum visibility for your research

Submit your manuscript at www.biomedcentral.com/submit

) Biomed Central 